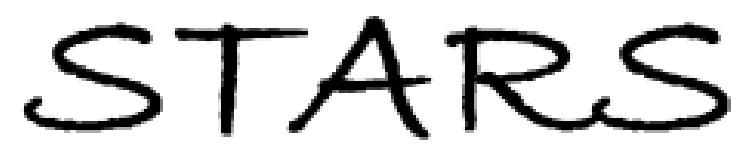

University of Central Florida

STARS

$1-1-2008$

\title{
Wide-view and single cell gap transflective liquid crystal display using slit-induced multidomain structures
}

Ruibo Lu

University of Central Florida

Zhibing Ge

University of Central Florida

Shin-Tson Wu

University of Central Florida

Find similar works at: https://stars.library.ucf.edu/facultybib2000 University of Central Florida Libraries http://library.ucf.edu

This Article is brought to you for free and open access by the Faculty Bibliography at STARS. It has been accepted for inclusion in Faculty Bibliography 2000 s by an authorized administrator of STARS. For more information, please contact STARS@ucf.edu.

\section{Recommended Citation}

Lu, Ruibo; Ge, Zhibing; and Wu, Shin-Tson, "Wide-view and single cell gap transflective liquid crystal display using slit-induced multidomain structures" (2008). Faculty Bibliography 2000s. 652.

https://stars.library.ucf.edu/facultybib2000/652

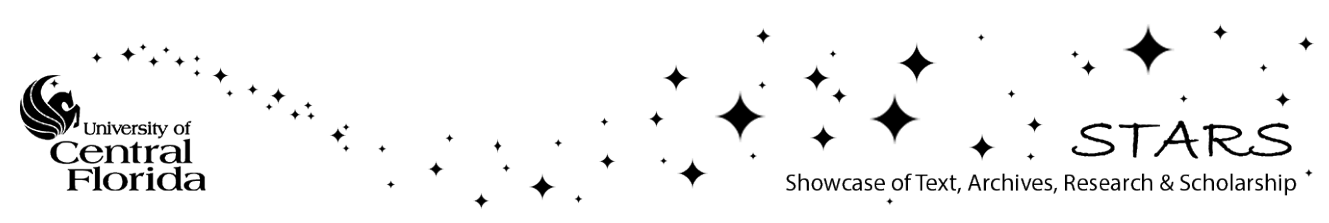




\section{Wide-view and single cell gap transflective liquid crystal display using slit-induced multidomain structures}

Cite as: Appl. Phys. Lett. 92, 191102 (2008); https://doi.org/10.1063/1.2926669

Submitted: 19 February 2008. Accepted: 21 April 2008. Published Online: 13 May 2008

Ruibo Lu, Zhibing Ge, and Shin-Tson Wu

\section{ARTICLES YOU MAY BE INTERESTED IN}

Wide-view transflective liquid crystal display for mobile applications

Applied Physics Letters 91, 231108 (2007); https://doi.org/10.1063/1.2822410

Transflective liquid crystal display using commonly biased reflectors

Applied Physics Letters 90, 22111 (2007); https://doi.org/10.1063/1.2745206

Electro-optic characteristics and switching principle of a nematic liquid crystal cell controlled by fringe-field switching

Applied Physics Letters 73, 2881 (1998); https://doi.org/10.1063/1.122617

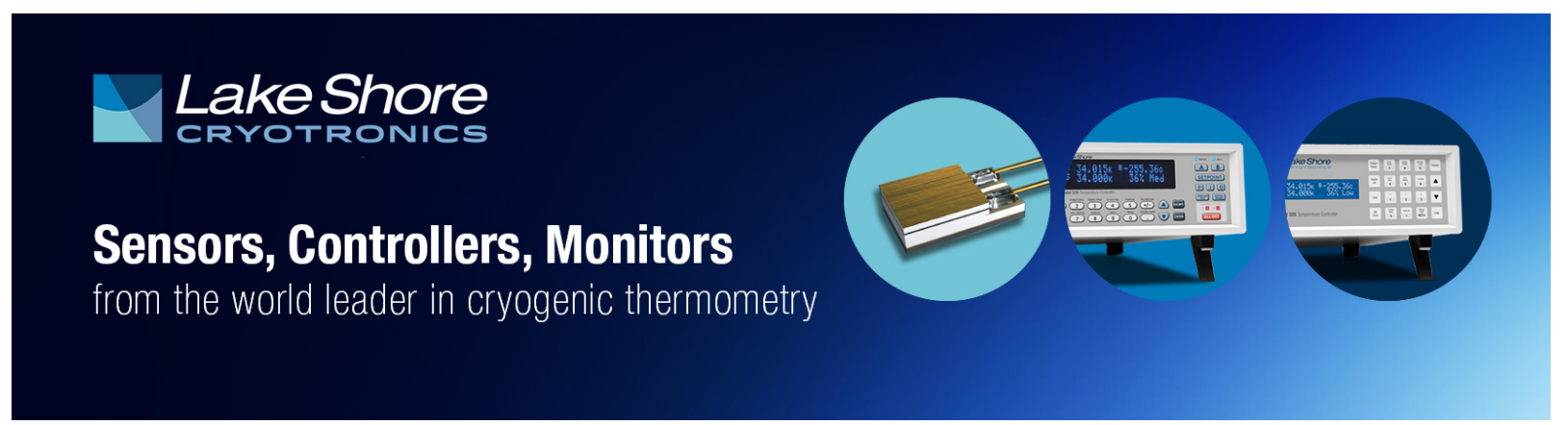




\title{
Wide-view and single cell gap transflective liquid crystal display using slit-induced multidomain structures
}

\author{
Ruibo Lu, Zhibing Ge, and Shin-Tson Wua) \\ College of Optics and Photonics, University of Central Florida, Orlando, Florida 32816, USA
}

(Received 19 February 2008; accepted 21 April 2008; published online 13 May 2008)

\begin{abstract}
A wide-view transflective liquid crystal display (TR-LCD) using a single cell gap multidomain vertical alignment (MVA) cell is proposed. Unlike a conventional MVA which uses physical protrusions to form multidomain structures, our TR-LCD uses slit-induced longitudinal and fringe electric fields to generate multidomains. As a result, wide-view, high contrast ratio, and high transmittance are obtained. By varying the slit density in the transmissive and reflective regions, the optical path-length disparity between these regions can be balanced. Potential application of this TR-LCD for mobile displays is emphasized. (C) 2008 American Institute of Physics.
\end{abstract}

[DOI: $10.1063 / 1.2926669$ ]

Transflective liquid crystal displays (TR-LCDs) have been widely used for mobile displays because of their sunlight readability. Some general requirements for a TR-LCD are wide viewing angle, high optical efficiency, high contrast ratio, low power consumption, and good color performance. To meet these challenges, several LC configurations, e.g., twisted-nematic, ${ }^{1,2}$ homogeneous alignment, ${ }^{3}$ vertical alignment (VA), ${ }^{4}$ in-plane switching, ${ }^{5,6}$ and fringe field switching ${ }^{7}$ have been proposed. Among these approaches, TR-LCDs can be further divided into double cell gaps versus single cell gap, and single thin film transistor (TFT) versus double TFTs. ${ }^{8}$ Presently, most TR-LCDs adopt the double cell gap approach for two reasons: (1) both transmissive $(T)$ and reflective $(R)$ modes can achieve maximum light efficiency, and (2) the gamma curve matching between the voltagedependent transmittance (VT) and reflectance (VR) is almost perfect. However, two shortcomings are found: (1) the $T$ region has a slower response time than the $R$ region because its cell gap is about twice thicker than that of the $R$ region, and (2) the viewing angle is relatively narrow, especially when the homogeneous cells are employed. ${ }^{9}$ To widen viewing angle, a special rodlike LC polymeric compensation film has to be used. ${ }^{10}$

Film-compensated multidomain vertical alignment (MVA) cells offer an excellent contrast ratio and wide viewing angle, and have been extensively used in LCD TVs. ${ }^{11,12}$ Both double and single cell gaps, wide-view TR-LCDs using MVA have been demonstrated. ${ }^{13,14}$ In the single cell gap approach, two reflective regions are required in order to match the VR with VT curves. Besides the fabrication complexity of such a device structure, a MVA TR-LCD has an intrinsic problem: It uses physical protrusions to form multiple domains. The light leakage from the protrusion edges degrades the contrast ratio and the dead zones above the opaque protrusions in the voltage-on state lower the optical efficiency. These problems become more pronounced in small sized LCD panels because of the smaller aperture ratio and increased protrusion density.

In this paper, we demonstrate a wide-view, single cell gap TR-LCD using MVA in which multidomain structures are formed by slit-induced longitudinal and fringe electric

\footnotetext{
${ }^{a)}$ Electronic mail: swu@ mail.ucf.edu.
}

fields. Without using physical protrusions, this MVA TRLCD exhibits a high optical efficiency, high contrast ratio, and wide viewing angle. Moreover, by optimizing the slit width and gap, the VT and VR curves reasonably match well.

Figure 1 shows a typical unit pixel and device configuration of the proposed MVA TR-LCD between two crossed circular polarizers. Each unit pixel is divided into $T$ and $R$ subpixels. As shown in Fig. 1(a), transparent indium tin oxide (ITO) layer is first deposited on the inner surface of the bottom glass substrate. Then, a portion of the ITO layer is etched off and covered with a metal layer such as aluminum (Al) to form bumpy reflector for the $R$ mode. The region not covered by the reflector works as $T$ mode. The $\mathrm{Al}$ reflector and ITO are connected to form pixel electrode which is connected to the same TFT. Therefore, the $R$ and $T$ subpixels are driven by a single TFT while keeping the same cell gap.

On the top substrate, some slits on the $R$ and $T$ regions are formed in the ITO common electrode. Here, the reflective opening ratio $\left(\mathrm{OR}_{R}\right)$ is defined as the effective slit projection

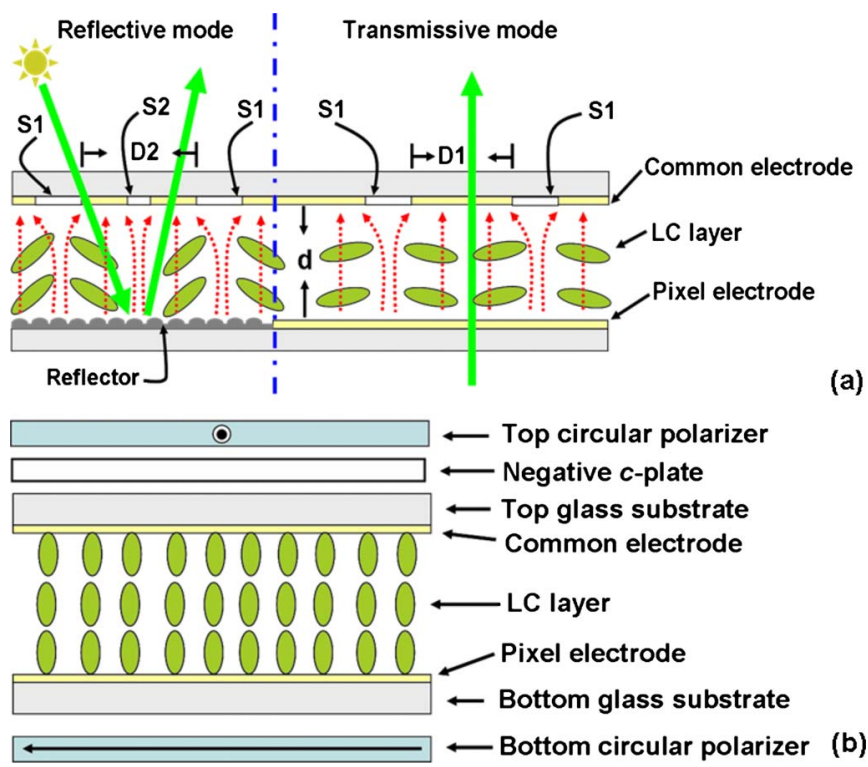

FIG. 1. (Color online) (a) The unit pixel and (b) device configuration of the proposed VA TR-LCD with crossed circular polarizers. The alignment layers are not shown here. 
area corresponding to that of the bottom $R$ region, and transmissive opening ratio $\left(\mathrm{OR}_{T}\right)$ as the effective slit projection area corresponding to that of the bottom $T$ region. The opening ratio is mainly determined by the slit shape, size, and the neighboring slit gap, $D 1$ and $D 2$, where the slit gap is usually larger than the slit width, $S 1$ and $S 2$, and the cell gap, $d$. The inner sides of both top and bottom substrates have a thin polyimide film (not shown) to align the LC molecules.

As Fig. 1(b) shows, the LC directors are vertically aligned on the substrate surfaces without rubbing process and the cell is in the normally black mode under crossed circular polarizers. When the applied voltage exceeds a threshold, the fringe electric fields surrounding the slits are generated while the longitural electric fields mainly exist inbetween the flat common and pixel ITO electrodes, as shown in Fig. 1(a), where the (red) dashed lines with arrows represent the electric field directions. The LC (with $\Delta \varepsilon<0$ ) directors would be reoriented perpendicular to the electric fields. Therefore, the backlight transmits through the crossed circular polarizers in the $T$ mode, and the ambient light is reflected by the bumpy reflectors and exits through the top circular polarizer in the $R$ mode. The longitudinal electric fields play the primary role to bend the LC directors. However, this directors bending is also guided by the fringe fields nearby the slit edges which force the LC directors to tilt to different directions. As a result, multidomains, e.g., four domains, are formed in a unit pixel when zigzag electrodes are configured. ${ }^{11,15}$

To obtain maximum transmittance and reflectance, the effective phase retardation in the $T$ region and $R$ region should satisfy the following relationship: $(d \Delta n)_{\mathrm{eff}, T}$ $=2(d \Delta n)_{\mathrm{eff}, R} \approx \lambda / 2$, where $\Delta n$ is the LC birefringence and $\lambda$ is the wavelength of the incident light. It is possible to tune the phase retardation by varying the opening areas (or slit density) as Fig. 1 shows. Because the ambient light traverses the LC layer twice in the $R$ region, we should design the slits to have $\mathrm{OR}_{R}>\mathrm{OR}_{T}$ in order to reduce the effective LC retardation in this region. Correspondingly, the longitudinal electric fields in the $R$ region are weaker than those in the $T$ region. The less reoriented LC directors in the $R$ region lead to a smaller phase retardation. However, because of double pass, its resultant phase retardation is similar to that of the $T$ region. Thus, a good match between VT and VR curves can be achieved.

Let us take one example to illustrate the design principle and device performance. Here, we select the repeated unit pixel size of the strip-shaped structure at $75 \times 25 \mu \mathrm{m}^{2}$, and choose the slit width $S 1=4 \mu \mathrm{m}$ and slit gap $D 1=18 \mu \mathrm{m}$ for the $T$ region, and slit gap $D 2=12 \mu \mathrm{m}$ for the $R$ region. A guiding slit $S 2$ whose width is $2 \mu \mathrm{m}$ is located at the center of $D 2$ in the $R$ region to avoid the domain corruption in the voltage-on state. Correspondingly, the reflective opening ratio $\mathrm{OR}_{R}$ is $31 \%$ and the $\mathrm{OR}_{T}$ is $18 \%$. The cell gap between the top and bottom substrates is $4 \mu \mathrm{m}$. A negative LC mixture Merck MLC-6608 $(\Delta n=0.083$ at $\lambda=550 \mathrm{~nm}, \Delta \varepsilon=-4.2$ and rotational viscosity $\gamma_{1}=0.186 \mathrm{~Pa} \mathrm{~s}$ ) is vertically aligned to the substrates in the initial state. Its azimuthal angle is $0^{\circ}$ and the pretilt angle is $90^{\circ}$.

Figure 2 shows the simulated LC director distribution of the proposed transflective VA cell with the strip-shaped multislits at $V=5 \mathrm{~V}_{\mathrm{rms}}$ between the common and pixel electrodes. The distribution is the side view cut from the center of the pixel unit along the $Y$-axis direction. It is observed that

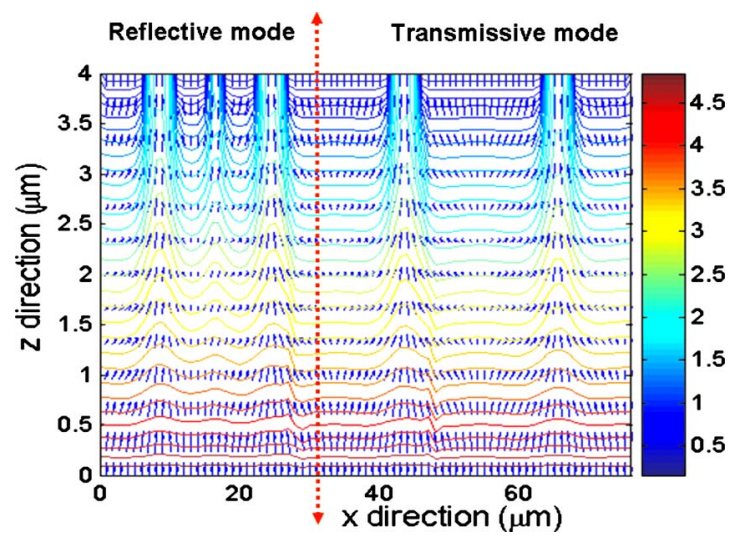

FIG. 2. (Color online) The simulated LC director distribution of the twodomain VA TR-LCD with strip-shaped multislits. The LC employed is Merck MLC-6608 and the applied voltage is $V=5 \mathrm{~V}_{\mathrm{rms}}$.

the LC directors are reoriented perpendicular to the electric fields due to the combined effect from the fringing field and the longitural electric field in both $R$ and $T$ parts. Therefore, in a voltage-on state a two-domain VA TR-LCD is formed from the strip-shaped multislits in each unit pixel.

Figure 3 is a plot of VT and VR curves of the transflective VA cell with the strip multislits under crossed circular polarizers at $\lambda=550 \mathrm{~nm}$. The $T$ and $R$ parts have the same threshold voltage at $2.25 \mathrm{~V}_{\text {rms }}$. Both VT and VR curves show a high transmittance/reflectance at $5 \mathrm{~V}_{\text {rms }}$ (a typical TFT driving voltage), which is $30.2 \%$ for the $T$ region and $30.1 \%$ for the $R$ region. The employed circular polarizer pair has a maximum transmittance of $35 \%$. Therefore, the strip-shaped multislit transflective VA cell has a high normalized optical efficiency of $86 \%$ for both $T$ and $R$ regions at $5 \mathrm{~V}_{\text {rms }}$.

From the inset in Fig. 3, the VT and VR curves fairly match well when the applied voltage is below $5 \mathrm{~V}_{\text {rms. }}$. To quantitatively characterize the gamma curve matching between the VT and VR curves, the root-mean-square (rms) value of the difference between the sampled transmittance and reflectance values is evaluated using rms $=\sqrt{\left(\sum_{i=1}^{N}\left(T_{i}-R_{i}\right)^{2}\right) / N}$, where $N$ is the number of sample points, $T_{i}$ the transmittance, and $R_{i}$ the reflectance at a given

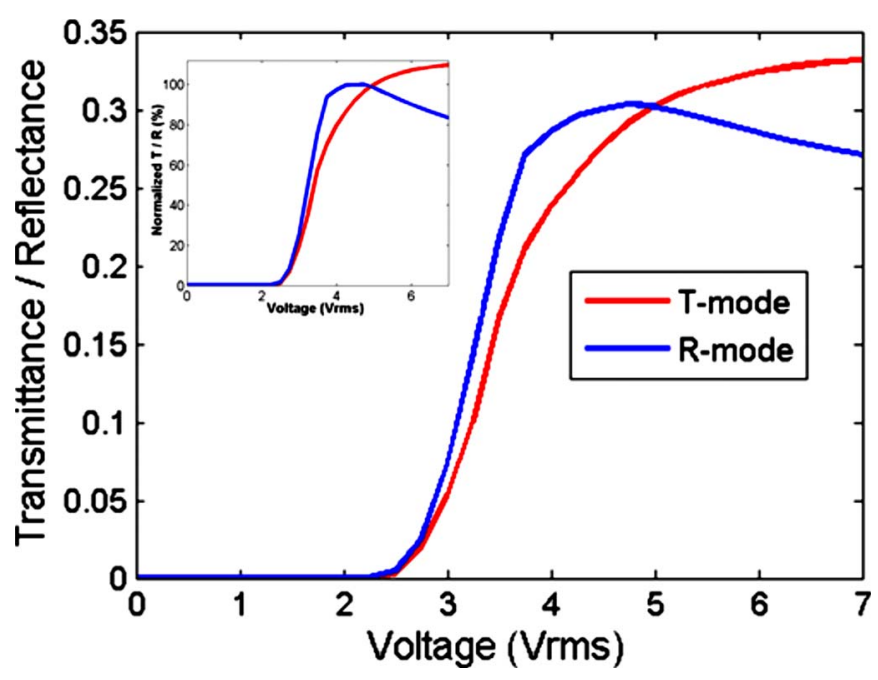

FIG. 3. (Color online) The VT and VR curves of the two-domain VA TRLCD with the strip-shaped multislits under crossed circular polarizers. The LC material is MLC- 6608 and $\lambda=550 \mathrm{~nm}$. The inset shows the normalized VT and VR curves. 

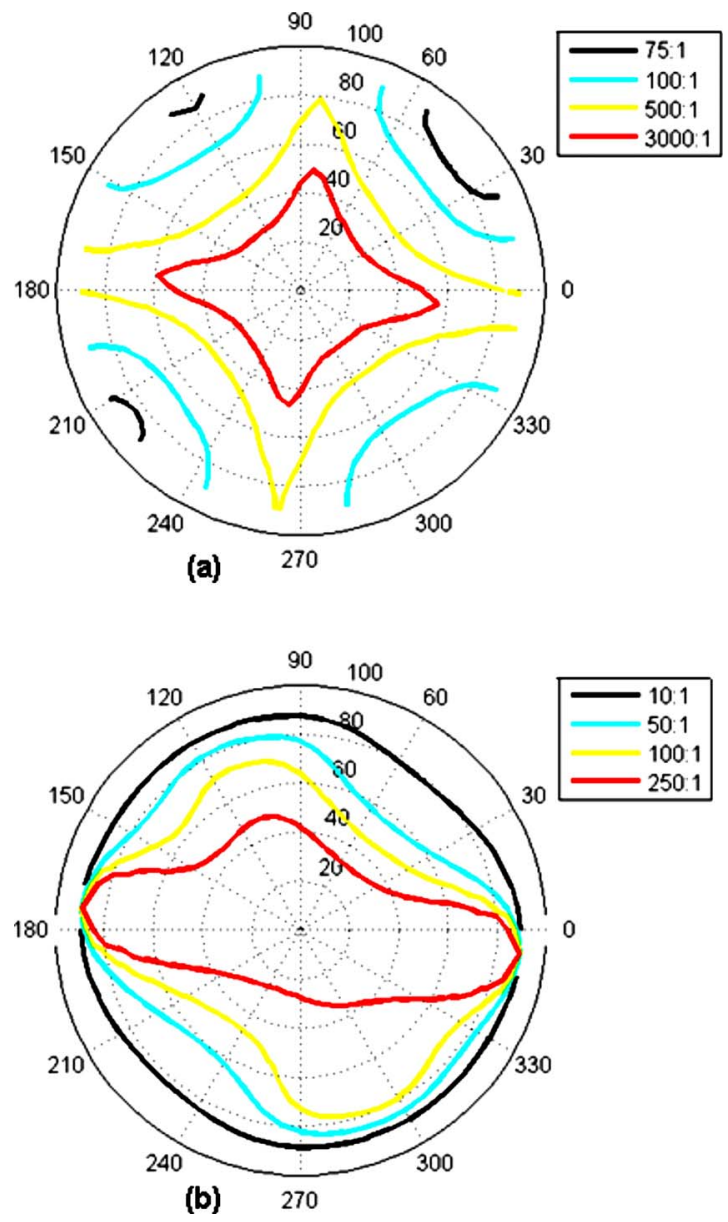

FIG. 4. (Color online) The isocontrast contour of the two-domain VA TRLCD between 0 and $5 \mathrm{~V}_{\text {rms }}$ : (a) $T$ mode and (b) $R$ mode. A white light source, two wide-view circular polarizers, and a negative $c$-plate with $d \Delta n=-332 \mathrm{~nm}$ are used for calculations.

voltage $V_{i}$. Based on this definition, a smaller rms value implies to a better match between the VT and VR curves. For the VT and VR curves shown in Fig. 3, by sampling $N$ $=256$ voltage points between $V=0 \mathrm{~V}_{\mathrm{rms}}$ (corresponding to gray level 0 ) and $V=5 \mathrm{~V}_{\text {rms }}$ (gray level 255), the calculated rms difference between the VR and VT curves is $\sim 4 \%$. It indicates that the TR-LCD can be driven by a single TFT while possessing the matched VT and VR curves.

It is commonly known that optical phase compensation films are required in order to reduce the light leakage at oblique angles of a VA LC cell for achieving wide viewing angle. ${ }^{16}$ In our simulations, we use the wide-view circular polarizers ${ }^{17}$ which consist of a series of uniaxial compensation films to work as broadband circular polarizers for widening the viewing angle of the two-domain VA TR-LCD, as Fig. 1(b) shows. A negative $c$-plate whose $d \Delta n=-332 \mathrm{~nm}$ is added after the first wide-view circular polarizer to cancel the off-axis light leakage from the LC layer. The contrast ratio is calculated between 0 and $5 \mathrm{~V}_{\text {rms }}$ under a white light source mixed with the red, green, and blue primaries at 3:6:1 weighting ratios.

Figure 4(a) is a plot of the viewing angle of the TR-LCD in the $T$ region. The isocontrast contour exceeds 3000:1 within the $\pm 30^{\circ}$ viewing cone. Within the $\pm 80^{\circ}$ view cone, the contrast ratio keeps higher than $75: 1$. As for the $R$ region, the 250:1 isocontrast contour line almost covers the whole horizontal directions, as shown in Fig. 4(b), but the viewing cone shrinks to $30^{\circ}$ at the $45^{\circ} / 225^{\circ}$ direction. For the $10: 1$ contrast ratio, the viewing cone is as wide as $\pm 80^{\circ}$. This demonstrates that the proposed TR-LCD has a high contrast ratio and wide-view angle in both $T$ and $R$ modes. These properties are especially desirable for high performance mobile displays.

In conclusion, we have proposed a wide-view single cell gap TR-LCD which utilizes the slit-induced fringe and longitudinal electric fields to form multiple LC domains without using physical protrusions. This TR-LCD exhibits a high optical efficiency, high contrast ratio, wide viewing angle in both $T$ and $R$ regions, and matched gamma curves. Its application for mobile LCDs is foreseeable.

The authors are indebted to the financial support from Chi-Mei Optoelectronics, Taiwan.

${ }^{1}$ M. Schadt and W. Helfrich, Appl. Phys. Lett. 18, 127 (1971).

${ }^{2}$ S. T. Wu and C. S. Wu, Appl. Phys. Lett. 68, 1455 (1996).

${ }^{3}$ M. Shibazaki, Y. Ukawa, S. Takahashi, Y. Iefuji, and T. Nakagawa, SID Int. Symp. Digest Tech. Papers 34, 90 (2003).

${ }^{4}$ Z. Ge, X. Zhu, R. Lu, T. X. Wu, and S. T. Wu, Appl. Phys. Lett. 90, 221111 (2007).

${ }^{5}$ O. Itou, S. Hirota, Y. Sekiguchi, S. Komura, M. Morimoto, J. Tanno, K. Fukuda, T. Ochiai, H. Imayama, T. Nagata, and T. Miyazawa, SID Int. Symp. Digest Tech. Papers 37, 832 (2006).

${ }^{6}$ R. Lu, Z. Ge, Q. Hong, and S. T. Wu, J. Disp. Technol. 3, 15 (2007).

${ }^{7}$ J. H. Song, Y. J. Lim, M.-H. Lee, S. H. Lee, and S. T. Shinb, Appl. Phys. Lett. 87, 011108 (2005)

${ }^{8}$ X. Zhu, Z. Ge, T. X. Wu, and S. T. Wu, J. Disp. Technol. 1, 15 (2005).

${ }^{9}$ S. T. Wu and C. S. Wu, Liq. Cryst. 24, 811 (1998).

${ }^{10}$ T. Uesaka, S. Ikeda, S. Nishimura, and H. Mazaki, SID Int. Symp. Digest Tech. Papers 38, 1555 (2007).

${ }^{11}$ Y. Koike and K. Okamoto, Fujitsu Sci. Tech. J. 35, 221 (1999).

${ }^{12}$ Q. Hong, T. X. Wu, X. Zhu, R. Lu, and S. T. Wu, Appl. Phys. Lett. 86, 121107 (2005)

${ }^{13}$ S. H. Lee, H. W. Do, G. D. Lee, T. H. Yoon, and J. C. Kim, Jpn. J. Appl. Phys., Part 2 42, L1455 (2003).

${ }^{14}$ C. Y. Tsai, M. J. Su, C. H. Lin, S. C. Hsu, C. Y. Chen, Y. R. Chen, Y. L. Tsai, C. M. Chen, C. M. Chang, and A. Lien, Proceedings of the Asia Display, 2007 (unpublished), Vol. 1, p. 24.

${ }^{15}$ R. Lu, X. Zhu, S. T. Wu, Q. Hong, and T. X. Wu, J. Disp. Technol. 1, 3 (2005).

${ }^{16}$ D. K. Yang and S. T. Wu, Fundamentals of Liquid Crystal Devices (Wiley, New York, 2006).

${ }^{17}$ Q. Hong, T. X. Wu, X. Zhu, R. Lu, and S. T. Wu, Opt. Express 13, 8318 (2005). 